Rev. Int. Contam. Ambie. 37, 133-143, 2021

https://doi.org/10.20937/RICA.53714

\title{
RESIDUOS DE PLAGUICIDAS EN BRÓCOLI (Brassica oleracea VAR. ITÁLICA) PARA EL MERCADO NACIONAL E INTERNACIONAL
}

\author{
PESTICIDES RESIDUES IN BROCCOLI (Brassica oleracea var. Italica) for the national and international market
}

\begin{abstract}
Diego Francisco CASTRO-VALDEZ ${ }^{1}$, Mario PÉREZ-GRAJALES ${ }^{1}$, Ma. Antonia PÉREZ-OLVERA ${ }^{2 *}$, Luis Fernando CONTRERAS-CRUZ ${ }^{1}$ y Joel PINEDA-PINEDA ${ }^{2}$
\end{abstract}

${ }^{1}$ Departamento de Fitotecnia, Instituto de Horticultura, Universidad Autónoma Chapingo, km 38.5 Carretera Federal México-Texcoco, 56230 Chapingo, Estado de México, México.

${ }^{2}$ Posgrado en Agroecología y Sustentabilidad, Colegio de Postgraduados, Carretera Federal México-Texcoco, 56230 Texcoco, Estado de México, México.

${ }^{3}$ Departamento de Suelos, Universidad Autónoma Chapingo, km 38.5 Carretera Federal México-Texcoco, 56230 Chapingo, Estado de México, México.

*Autora para correspondencia: molvera@colpos.mx

(Recibido: agosto de 2019; aceptado: agosto de 2020)

Palabras clave: UHPLC-MS/MS, LMR, residuos de plaguicidas, calidad de producto.

\begin{abstract}
RESUMEN
La investigación tuvo como objetivo analizar las características agronómicas y la presencia de residuos de plaguicidas en cabezuela de brócoli (Brassica oleracea var. Itálica) listas para su comercialización en dos sistemas de producción, uno para el mercado nacional y otro para exportación. Se analizaron 78 muestras provenientes de un empaque de Irapuato, Guanajuato, México, y de la Central de Abasto de Huixcolotla, Puebla, México. Para la evaluación agronómica se consideraron las siguientes variables: rendimiento peso, diámetro y color de la cabezuela. El análisis de residuos de plaguicidas se realizó mediante el método 2007.01 de la AOAC modificado, usando cromatografía líquida de ultra alta eficiencia acoplada a espectrometría de masas en tándem (UHPLC-MS/MS) como técnica de separación y cuantificación. Los valores medios de diámetro y peso de cabezuela fueron de $15.13 \mathrm{~cm}$ y $464.95 \mathrm{~g}$ para Guanajuato y de $13.67 \mathrm{~cm}$ y $539.47 \mathrm{~g}$ para Puebla, respectivamente. El diámetro y peso de la cabezuela cumplen con los estándares para el mercado nacional. Los resultados de los análisis de residuos de plaguicidas indican que, de las 78 muestras, 42 resultaron libres de plaguicidas, 14 con concentraciones no cuantificables (trazas) y 22 con al menos un residuo. Los plaguicidas encontrados fueron boscalid $(0.0061 \mathrm{mg} / \mathrm{kg})$, dimetoato $(0.0068 \mathrm{mg} / \mathrm{kg})$, imidacloprid $(0.0087 \mathrm{mg} / \mathrm{kg})$, metamidofos $(0.0266 \mathrm{mg} / \mathrm{kg})$, ometoato $(0.0111 \mathrm{mg} / \mathrm{kg})$, penconazole $(0.0039 \mathrm{mg} / \mathrm{kg})$, propamocarb $(0.0061 \mathrm{mg} / \mathrm{kg})$ y thiametoxan $(0.0045 \mathrm{mg} / \mathrm{kg})$. Las concentraciones encontradas no rebasan los límites máximos de residuos establecidos por el Servicio Nacional de Sanidad, Inocuidad y Calidad Agroalimentaria y la Environmental Protection Agency.
\end{abstract}

Key words: UHPLC-MS/MS, MRL, pesticide residues, quality product. 


\begin{abstract}
The objective of this research was to analyze the agronomic characteristics and the presence of pesticides residues in broccoli heads ready for commercialization, in two production systems, one for the national market and other for export. Seventy-eight samples from a package of Irapuato, Guanajuato, Mexico and the supply center of Huixcolotla Puebla, Mexico were analyzed. For the agronomic evaluation, the following variables were considered: yield, weight, diameter, and color of the head. Pesticide residues were analyzed with the AOAC method 2007.01, modified by using ultra high efficiency liquid chromatography coupled to tandem mass spectrometry (UHPLC-MS/MS) as a separation and quantification technique. The mean values of head diameter and weight were $15.13 \mathrm{~cm}$ and $464.95 \mathrm{~g}$ for Guanajuato and $13.67 \mathrm{~cm}$ and $539.47 \mathrm{~g}$ for Puebla, respectively. These values comply with the standards for the national market. The results from the analysis of pesticides residues indicate that 42 of the 78 samples were free of pesticides, 14 samples had non-quantifiable concentrations (traces) and 22 samples contained at least one residue. The pesticide residues found were: boscalid $(0.0061 \mathrm{mg} / \mathrm{kg})$, dimethoate $(0.0068 \mathrm{mg} / \mathrm{kg})$, imidacloprid $(0.0087 \mathrm{mg} /$ $\mathrm{kg})$, methamidophos $(0.0266 \mathrm{mg} / \mathrm{kg})$, ometoate $(0.0111 \mathrm{mg} / \mathrm{kg})$, penconazole $(0.0039$ $\mathrm{mg} / \mathrm{kg})$, propamocarb $(0.0061 \mathrm{mg} / \mathrm{kg})$, and thiametoxan $(0.0045 \mathrm{mg} / \mathrm{kg})$. In none of the cases the found concentrations exceed the maximum residues limits established by the National Service for Agro-Alimentary Public Health, Safety and Quality, and the Environmental Protection Agency.
\end{abstract}

\section{INTRODUCCIÓN}

Los cultivos de crucíferas son importantes en México. Se siembran alrededor de 50000 ha, de las cuales $74.42 \%$ son de brócoli, $12.45 \%$ de col y 7.0 $\%$ de coliflor. Se cultivan en suelos ricos en materia orgánica, en climas frescos y húmedos. Desde el punto de vista nutricional, aportan grandes cantidades de minerales, proteínas y vitaminas (Suárez et al. 2006). La producción de brócoli se inició en México a consecuencia de la popularidad que gozaba en otros países, principalmente en EUA, y como una oportunidad comercial ante la capacidad de producirlo a menor costo (Rocha y Cisneros 2019). El auge del brócoli en México se dio a partir de 1970 y es atribuido a la rentabilidad del cultivo y a los nuevos hábitos de consumo sano (Bujanos et al. 2013, Garay y Chávez 2014).

Guanajuato es el principal productor de brócoli en México, con el $62 \%$ del total de la superficie cosechada, el $57.8 \%$ de la producción total y el $46 \%$ del valor de ésta, con un rendimiento medio de 12.98 t/ha (Rocha y Cisneros 2019).

E1 $95 \%$ del brócoli procesado se destina a la exportación, siendo EUA el principal destino (98 \% de las exportaciones). Otros clientes menores son Japón y algunos países de la Unión Europea. La producción y comercialización de hortalizas para la exportación directa hacia EUA, Canadá y Cuba se han incrementado. En 2003 se exportaron más de 2600 embarques anuales con más de $30000 \mathrm{t}$ de hortaliza (Fernández-Cevada y Vázquez-Ortiz 2003). Para 2019 se reportó un volumen de producción de 270 939 t de brócoli, de las cuales se exportó alrededor del $70 \%$ de acuerdo con datos del Servicio de Información Agroalimentaria y Pesquera (SIAP 2020).

Otros estados importantes para la producción de brócoli en México son Michoacán, Jalisco, Sonora, Puebla y Baja California (Rocha y Cisneros 2019). En el estado de Puebla, el brócoli se siembra en 24 municipios, ocupando un área de 1202 ha; destaca el distrito de riego de Valsequillo, con siete municipios. La producción total se comercializa en el mercado de Huixcolotla, Puebla.

La producción de brócoli se ve afectada por varias especies de lepidópteros, cuyas larvas causan daños considerables al cultivo. Suárez et al. (2006) mencionan como principal daño la contaminación del producto final o bien la presencia de pulgones en la cabezuela, además de la defoliación y el daño a las hojas, que deja visibles peciolos y nervaduras en éstas (Marín y Bujanos 2001). Entre las especies más importantes están el gusano del corazón de la col (Copitarsia consueta), el gusano falso medidor (Trichoplusia ni) y la palomilla blanca de la col (Leptophobia aripa).

Los plaguicidas cumplen con una función primordial en el control de numerosos insectos, hongos, malezas y otros organismos que compiten con el hombre por productos agropecuarios y forestales 
(Lagunes-Tejeda y Villanueva-Jiménez 1994). Sin embargo, en los últimos años se ha incrementado la preocupación por las consecuencias de su uso. La exposición crónica a ciertos plaguicidas se ha asociado con afectaciones a la salud - como el cáncer-en algunos sitios, particularmente aquellos con actividad agrícola (Wigle et al. 2007, Weichenthal et al. 2010). Pérez et al. (2009) mencionan los riesgos hacia la salud humana generados por la manipulación, aplicación y exposición ocupacional de plaguicidas. Otro aspecto importante es el consumo de alimentos con residuos de estas sustancias. Aun cuando es conocido que los plaguicidas son agentes químicos tóxicos, también se sabe que sus beneficios hacia la sociedad son grandes, ya que han permitido potencializar la economía en términos de mejoras en el volumen de la producción agrícola a nivel mundial, mediante la disminución de plagas y vectores (Martínez et al. 2012). La presencia de residuos de plaguicidas en los alimentos se debe, principalmente al uso inadecuado o indiscriminado de éstos en la agricultura. La eliminación de plaguicidas depende de diversos factores, como el crecimiento propio del vegetal, la acción de agentes atmosféricos, el grado de solubilidad y volatilidad del plaguicida, el tipo de degradación química y la naturaleza del propio plaguicida (Ortiz et al. 2003). Otro factor importante es el intervalo de seguridad (días entre la aplicación y la cosecha del producto) recomendado para cada plaguicida por la Comisión Intersecretarial para el Control del Proceso y Uso de Plaguicidas y Sustancias Tóxicas (CICOPLAFEST 2004).

Las normas establecidas en cuanto a la inocuidad de los alimentos y a los niveles máximos de residuos (LMR) en los productos básicos se han hecho más estrictas. Se han establecido nuevas leyes, como la Directiva Europea 91/414/CEE en la Unión Europea (UE) y la Ley de Protección de la Calidad (FQPA) en EUA, que incrementó los estándares para la protección de la salud humana, los trabajadores y el medio ambiente. Las normas de calidad incluyen la reevaluación permanente de los LMR (Ferrer et al. 2005).

Los efectos negativos sobre la salud humana que se han producido como consecuencia de la exposición a plaguicidas $\mathrm{u}$ otros factores ambientales pueden expresarse inmediatamente o tardar años en manifestarse. Estos últimos deben ser de mayor atención para identificar el problema, antes de la aparición de los síntomas y antes de que se manifiesten los efectos adversos (Jaga y Dharmani 2005, Martínez et al. 2012).

La calidad del brócoli es muy importante para los distribuidores y consumidores. El tamaño del florete es el principal carácter comercial en esta hortaliza (Westcott y Callan 1990), seguido de otros indicadores como floretes cerrados, color verde oscuro brillante y cabezas compactas (Cantwell y Suslow 2007). El manejo agrícola del cultivo de brócoli debe estar encaminado a desarrollar las características de tamaño, peso, forma, color y aspecto de la cabezuela (Soto 1991). Adicionalmente el brócoli para el mercado orgánico debe estar libre de residuos de plaguicidas y en el caso de brócoli para exportación, el producto no debe rebasar los LMR establecidos por la Environmental Protection Agency (EPA 2019) y por el Servicio Nacional de Sanidad e Inocuidad Agroalimentaria (SENASICA).

El objetivo de esta investigación fue analizar las características agronómicas del brócoli (Brassica oleracea var. Italica) y la presencia de residuos de plaguicidas en cabezuelas listas para su comercialización en dos sistemas de producción (el mercado nacional y el de exportación).

\section{MATERIALES Y MÉTODOS}

\section{Muestreo y manejo de muestras}

Se tomaron 78 muestras, de las cuales 70 provenían de comunidades de los tres principales municipios que abastecen de brócoli a la central de abastos de Huixcolotla, Puebla (Los Reyes de Juárez, Palmar de Bravo y Quecholac). Los sitios de donde provenían las muestras fueron Reyes de Juárez, La Concordia, San José Tuzuapán, San Bartolomé Coscomeya, Palmar de Bravo, Palmarito, San Miguel Xaltepec, Quecholac, Jesús de Nazareno, Froylán C. Manjarrez y Benito Juárez. El brócoli que se comercializa en la central de abasto es destinado al mercado nacional. Las ocho muestras restantes fueron tomadas de un empaque de brócoli para exportación a EUA en la ciudad de Irapuato, Guanajuato, con brócoli proveniente de los municipios de Abasolo, León, Valle de Santiago e Irapuato.

Se tomaron 70 muestras (de 10 cabezuelas cada una) de las camionetas cargadas con brócoli que llegan al área de subastas de la central de abasto: 15 del municipio de Los Reyes de Juárez, 40 de Palmar de Bravo y 15 de Quecholac. Las cabezuelas se colocaron en bolsas de plástico para ser transportadas a las instalaciones de Laboratorios GISENA, ubicados en San Luis Huexotla, Texcoco, Estado de México, donde se colocaron en cámaras de refrigeración (temperatura de $4{ }^{\circ} \mathrm{C}$ ). Posteriormente se realizaron la toma de datos y los análisis de residuos de plaguicidas. 
En el empaque de brócoli de Irapuato, Guanajuato, se aplicó la técnica de muestreo por conveniencia, debido a que no se nos permitió la entrada al áreade empaque para realizar los muestreos, los encargados llevaban las muestras a las oficinas y ahí se tomaban los datos. Las muestras se tomaron en bolsas plásticas y se colocaron en hieleras de unicel con bolsas de gel congelado (sustituto de hielo) para su traslado al laboratorio de residuos tóxicos en vegetales de Laboratorios GISENA.

\section{Variables de calidad física}

El peso de las cabezuelas se cuantificó con una balanza digital, en tanto que el diámetro ecuatorial de la cabezuela de brócoli se midió con un vernier. El color se determinó utilizando una tabla de colores de la Royal Horticultural Society (2007), la cual incluye cuatro grupos de tarjetas agrupadas en abanico, correspondientes a cuatro gamas de colores: amarillo al rojo, rojo-púrpura al azul, azul-verdoso al amarillo-verdoso y grisáceos de todos los grupos de color. El color queda descrito por un número y una letra (Pérez et al. 2008). El rendimiento se calculó con el peso promedio de las cabezuelas y la densidad de población. Se consideró una densidad de población media de 50000 plantas/ha. A las variables diámetro y peso de cabezuela, así como al rendimiento, se les realizó un análisis de varianza y prueba de comparación de medias Tukey $(\mathrm{p}=0.05)$ para determinar si existen diferencias significativas en el brócoli para el mercado internacional y el nacional representados por las dos zonas estudiadas.

\section{Residuos de plaguicidas}

Para el análisis de residuos de plaguicidas se tomó como referencia el método 15662 establecido por la Comité Europeo de Normalización (CEN 2009), que es una modificación del método 2007.01 de AOAC International: Residuos de plaguicidas en alimentos por extracción de acetonitrilo y partición con sulfato de magnesio (Anastassiades et al. 2003). Se utilizaron $500 \mathrm{~g}$ de material vegetal, el cual fue molido en licuadora hasta formar una pasta homogénea. El recipiente donde se molió el material se lavó con hexano, acetona y agua destilada.

Las muestras molidas se guardaron en recipientes de plástico en condiciones de congelación. Se tomaron $10 \mathrm{~g}$ de pasta en un tubo falcón de $50 \mathrm{~mL}$ y se agregaron $10 \mathrm{~mL}$ de acetonitrilo, $100 \mu \mathrm{L}$ del estándar interno (atrazina, $0.01 \mathrm{mg} / \mathrm{L}$ ) y las sales de extracción (1.5 g de acetato sódico y $6 \mathrm{~g}$ de $\mathrm{MgSO}_{4}$ ). La mezcla se agitó en un vórtex por $10 \mathrm{~min}$ y se centrifugó por $5 \mathrm{~min}$ a $3000 \mathrm{rpm}$. Se tomó $1 \mathrm{~mL}$ del sobrenadante colocándolo en otro tubo (tubo 2), con las sales de limpieza (150 mg de $\mathrm{MgSO}_{4}, 50 \mathrm{mg}$ de PSA); se agitó en un vórtex por 10 min y se centrifugó por $5 \mathrm{~min}$ a $3000 \mathrm{rpm}$. Se tomaron $200 \mu \mathrm{L}$ de este filtrado y se colocaron en un vial, adicionando $800 \mu 1$ de fase móvil A (formiato de amonio). El vial se lleva al vórtex por 3 min y se procedió a inyectar en el equipo. La evaluación se realizó mediante los estudios de recuperación.

Para llevar a cabo dichos estudios se realizó la fortificación de muestras blanco con una mezcla de 104 residuos de plaguicidas validados por el Laboratorio GISENA. Se utilizaron cinco niveles de concentración de $6.25,12.5,25,50$ y $100 \mu \mathrm{L}$. Se utilizó un UHPLC modelo Acquity acoplado a un espectro MS/MS modelo XEVO TQ-S (ambos de Waters Corporation), y una columna de cromatografía Acquity UPLC CSH C18 de $1.7 \mu \mathrm{m}$ con tamaño de partícula de $2.1 \mathrm{~mm}$ de diámetro interno y $50 \mathrm{~mm}$ de longitud.

Se realizó un análisis multirresiduo que incluyó 104 moléculas de plaguicidas, validado por el laboratorio de residuos de plaguicidas en vegetales. Dicho análisis es solicitado con fines de certificación orgánica y para productos destinados al mercado de exportación. La presencia de residuos de plaguicidas se determinó mediante los tiempos de retención de cada uno de los plaguicidas considerados para el análisis. Además, se consideró el límite de detección como la mitad de la concentración del analito presente en el nivel $1(6.25 \mu \mathrm{L})$ de la curva de calibración. El límite de cuantificación es la concentración más baja de un analito que se puede determinar con precisión aceptable (capacidad de repetición) y exactitud en las condiciones indicadas de la prueba (SENASICA 2016). Dicho límite establece un rango de recuperación medio de 70-120\%. Los porcentajes de desviación y recuperación son indicadores de precisión de los análisis.

\section{RESULTADOS Y DISCUSIÓN}

\section{Calidad física de las cabezuelas de brócoli Peso y diámetro de cabezuela}

El análisis estadístico realizado a la variable diámetro de la cabezuela de brócoli, presenta diferencias estadísticas significativas con valores de $15.13 \mathrm{y}$ $13.53 \mathrm{~cm}$ para Guanajuato y Puebla, respectivamente. La variable peso de cabezuela presenta valores medios de 539.47 y 464.95 g para Puebla y Guanajuato, respectivamente, con la misma tendencia en las muestras analizadas a nivel de sitio (Cuadro I). Las diferencias encontradas pueden atribuirse al uso de 
CUADRO I. PESO Y DIÁMETRO PROMEDIO DE CABEZUELAS DE BRÓCOLI DE LAS DOS REGIONES ESTUDIADAS (GUANAJUATO Y PUEBLA).

\begin{tabular}{|c|c|c|c|}
\hline Muestra & $\begin{array}{c}\text { Peso } \\
(\mathrm{g})\end{array}$ & Región & $\begin{array}{l}\text { Diámetro } \\
(\mathrm{cm})\end{array}$ \\
\hline Reyes de Juárez & $682.97 \mathrm{a}$ & Irapuato1 & $15.88 \mathrm{a}$ \\
\hline La Concordia & $582.43 \mathrm{ab}$ & León1 & $15.72 \mathrm{a}$ \\
\hline Tuzuapan & $564.35 \mathrm{ab}$ & Xaltepec & $14.33 b$ \\
\hline San Bartolo & $563.83 \mathrm{ab}$ & Abasolo1 & $13.97 \mathrm{bc}$ \\
\hline Palmar de Bravo & $542.78 b c$ & San Bartolo & $13.92 b c$ \\
\hline Irapuato1 & $539.63 \mathrm{bc}$ & Valle de Santiago 1 & $13.83 b c$ \\
\hline Palmarito & $536.24 \mathrm{bcd}$ & Tuzuapan & $13.82 b c$ \\
\hline Xaltepec & $530.56 \mathrm{bcd}$ & Palmar de Bravo & $13.75 b c$ \\
\hline Quecholac & $511.45 \mathrm{bcd}$ & Nazareno & $13.68 \mathrm{bc}$ \\
\hline Nazareno & $508.21 \mathrm{bcd}$ & Quecholac & $13.61 b c$ \\
\hline Manjarrez & $490.3 \mathrm{bcd}$ & Reyes de Juárez & $13.59 \mathrm{bc}$ \\
\hline Benito Juárez & $476.92 \mathrm{bcd}$ & La Concordia & $13.53 \mathrm{bc}$ \\
\hline Valle de Santiago 1 & $435.33 \mathrm{~cd}$ & Benito Juárez & $13.24 b c$ \\
\hline León1 & $428.28 \mathrm{~cd}$ & Palmarito & $13.21 b c$ \\
\hline Abasolo1 & $411.94 d$ & Manjarrez & $12.85 \mathrm{c}$ \\
\hline
\end{tabular}

${ }^{1}$ Muestras correspondientes al estado de Guanajuato (medias con la misma letra son estadísticamente iguales).

diferentes variedades o híbridos (con características genéticas específicas para cabezuela). Fraire et al. (2010) señalan que en los híbridos de brócoli existen diferencias de diámetro del florete, color (verde grisáceo a verde azuloso), tamaño y compactación de la cabezuela o pella, así como tamaño y consistencia de las flores que componen la inflorescencia, y presencia de tallo hueco (lo cual puede representar un problema cuando la producción se destina al mercado en fresco [Villalobos et al. 2005]). El diámetro de la cabezuela de brócoli es una característica importante cuando se comercializa en fresco (Pérez et al. 2014).

El diámetro ecuatorial de las cabezuelas, tanto de Guanajuato como de Puebla, se encuentra dentro de los estándares de alta calidad definidos en la norma mexicana NMX-FF-046-1982 (SEPAFIN 1983) y el pliego de condiciones para brócoli (PC-034-2005) para el uso de la marca oficial México Calidad Suprema (SE 2005). La norma NMX-FF-046-1982 establece que para la calidad México Extra y México 1 los brócolis deben presentar un diámetro ecuatorial mayor a $7 \mathrm{~cm}$, las yemas cerradas y la inflorescencia compacta. Fraire et al. (2010) reportan diámetros de florete de $17.1 \mathrm{a} 21 \mathrm{~cm}$ en las zonas de Apaseo el Grande, Guanajuato.

\section{Rendimiento}

Los rendimientos calculados de brócoli variaron de 20.6 a 34.1 t/ha. Los tres valores más bajos
(20.59, 21.4 y $21.7 \mathrm{t} / \mathrm{ha})$ corresponden a las muestras provenientes de Abasolo, León y Valle de Santiago, respectivamente. El comportamiento del rendimiento es congruente con el peso promedio de cabezuela, que fue menor en las muestras provenientes del estado de Guanajuato, a excepción de Irapuato, que tuvo un rendimiento de $26.9 \mathrm{t} / \mathrm{ha}$. En las comunidades de Puebla los rendimientos obtenidos van de 23.84 a 29.12 t/ha, sin embargo, destaca Los Reyes de Juárez con un rendimiento medio estimado de $34.15 \mathrm{t} / \mathrm{ha}$. Los rendimientos obtenidos presentan diferencias estadísticas significativas conformando cuatro grupos de medias (Fig. 1). En este estudio no se consideraron la selección de campo ni la clasificación de cabezuelas de brócoli, que regularmente se hace en los empaques o en las centrales de abasto. Villalobos et al. (2005) reportan que las maquiladoras de la región del Bajío clasifican las cabezuelas en grado 1 , grado 2 y desecho, y se pagan a precio diferenciado.

Los rendimientos calculados para las comunidades de Puebla son superiores a las reportadas en Tepeaca-Tecamachalco, Puebla, con un rendimiento comercial promedio de brócoli de $12 \mathrm{t} / \mathrm{ha}$, con una variación de 10 a 15 t/ha (Torres 2011). Para Guanajuato, Villalobos et al. (2005), reportan rendimientos de hasta $41 \mathrm{t} / \mathrm{ha}$, mediante el manejo de humedad a $20 \mathrm{kPa}$ de tensión.

\section{Color de las cabezuelas de brócoli}

En el cuadro II se presentan los resultados correspondientes a la caracterización del color de las cabezuelas de acuerdo con la tabla de colores de la Royal Horticultural Society (2007). Para tejidos vegetales, las muestras presentaron colores verde oscuro y verde azulado oscuro. Las muestras provenientes de siete de las comunidades de Puebla y dos de Guanajuato presentaron color verde oscuro y las muestras restantes presentaron coloración azul-verde oscura. La Norma Mexicana NMX-FF-046-1982 establece que el brócoli debe presentar un color verde o violeta grisáceo característico. Sin embargo, se ha determinado que la coloración del brócoli no afecta la calidad de la cabezuela (Shewfelt et al. 2006, Pérez et al. 2014). La variabilidad en la coloración está ligada al tiempo en que se realizó el corte y es un parámetro importante a considerar, sobre todo para el mercado en fresco (Pérez et al. 2014) y para el almacenamiento (Gnanasekharan et al. 1992). Los resultados obtenidos en color son similares a los reportados por Fernández et al. (2013) para los cultivares de brócoli Phartenon y Maratón, los cuales presentaron un color azul-verde. 


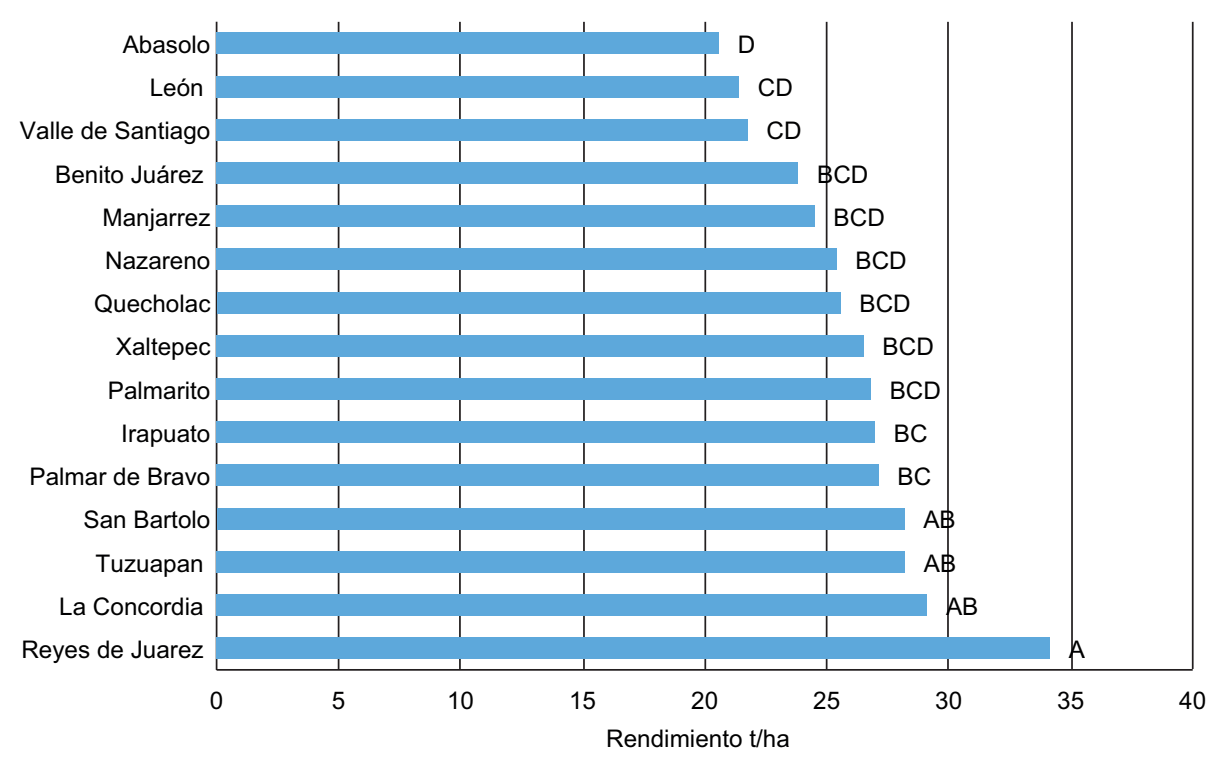

Fig. 1. Rendimiento estimado de brócoli en $\mathrm{t} / \mathrm{ha}$, de muestras provenientes de Guanajuato y Puebla.

CUADRO II. COLOR DE LAS CABEZUELAS DE BRÓCOLI DE ACUERDO CON LA TABLA DE COLORES DE LA ROYAL HORTICULTURAL SOCIETY (2007).

\begin{tabular}{cccc}
\hline Estado & Muestra & $\begin{array}{c}\text { RHS (grupo } \\
\text { verde) }\end{array}$ & Color \\
\hline \multirow{4}{*}{ La Concordia } & $133 \mathrm{a}$ & Azul verde oscuro \\
& Manjarrez & $131 \mathrm{a}$ & Azul verde oscuro \\
& Palmar de Bravo & $131 \mathrm{a}$ & Azul verde oscuro \\
& Tuzuapan & $131 \mathrm{a}$ & Azul verde oscuro \\
& Benito Juárez & $136 \mathrm{a}$ & Verde oscuro \\
& Nazareno & $136 \mathrm{a}$ & Verde oscuro \\
& Palmarito & $135 \mathrm{a}$ & Verde oscuro \\
& Quecholac & $135 \mathrm{a}$ & Verde oscuro \\
& Reyes de Juárez & $136 \mathrm{a}$ & Verde oscuro \\
& San Bartolo & $135 \mathrm{a}$ & Verde oscuro \\
& Xaltepec & $136 \mathrm{a}$ & Verde oscuro \\
\hline \multirow{4}{*}{ Guanajuato } & León & $133 \mathrm{a}$ & Azul verde oscuro \\
& Abasolo & $133 \mathrm{a}$ & Azul verde oscuro \\
& Irapuato & $135 \mathrm{a}$ & Verde oscuro \\
& Valle de Santiago & $136 \mathrm{a}$ & Verde oscuro \\
\hline
\end{tabular}

\section{Principales plagas del brócoli y su control}

Las principales plagas reportadas por los productores de brócoli que abastecen la central de abasto de Huixcolotla, Puebla, son, en orden de importancia, palomilla dorso de diamante (Plutella xylostella), pulgón cenizo (Brevicoryne brassicae), pulgon verde (Mysus persicae), gusano falso medidor (Trichoplusia $n i$ ) y palomilla blanca (Leptophobia aripa), que causan daños a los cultivos y afectan el rendimiento y la calidad. Para el control de las plagas se utilizan principalmente plaguicidas organofosforados, neonocotinoides, carbamatos, piretroides, y productos biológicos, los cuales tienen menor capacidad para acumularse en los tejidos, característica que les da ventaj, con relación a los plaguicidas organoclorados, los cuales salieron del mercado por ser altamente persistentes (Obare et al. 2010). Torres (2011) menciona que la palomilla dorso de diamante es reportada como la principal plaga en la región Tepeaca-Tecamachalco en conjunto con el gusano falso medidor; asimismo, menciona que los productos más utilizados para el control de plagas insectiles son Furadan (carbofurano, organofosforado), Arrivo (cipermetrina, piretroide) y Spintor (spinosad).

En el cuadro III se muestran los principales productos utilizados para el control de plagas de brócoli mencionados por productores de la zona de Tecamachalco, Puebla, el grupo químico al que pertenecen, su toxicidad y su clasificación mediante la lista actualizada de Pesticides Action Network International de plaguicidas altamente peligrosos (PAN 2019). Destaca el hecho de que siete de los ocho plaguicidas reportados están incluidos en la lista de plaguicidas altamente peligrosos.

\section{Análisis de la presencia de residuos de plaguicidas}

Los resultados indican que $42(53.8 \%)$ de las 78 muestras analizadas resultaron libres de plaguicidas y en el resto (36 muestras, $46.2 \%$ ) se encontraron 
CUADRO III. PRODUCTOS UTILIZADOS PARA EL CONTROL DE PLAGAS EN LA REGIÓN DE HUIXCOLOTLA, PUEBLA.

\begin{tabular}{|c|c|c|c|c|c|}
\hline $\begin{array}{l}\text { Nombre } \\
\text { comercial }\end{array}$ & $\begin{array}{l}\text { Nombre } \\
\text { común }\end{array}$ & Grupo químico & $\begin{array}{c}\text { Categoría de } \\
\text { riesgo de toxicidad }\end{array}$ & $\begin{array}{l}\text { Modo de } \\
\text { acción }^{2}\end{array}$ & PAP \\
\hline Belt & Flubendiamida & Diamida & I & 28 & Sí \\
\hline Spintor, Tracer & Spinosad & Spinosos & IV & 5 & Sí \\
\hline Furadan & Carbofuran & Carbamatos & I & 1 & Sí \\
\hline Arrivo & Cipermetrina & Piretroide & II & 3 & Sí \\
\hline Disparo, Foley & Clorpirifos & Organofosforado & I & 1 & Sí \\
\hline Muralla max & Imidacloprid & Neonicotinoide & II & 4 & Sí \\
\hline Vidate & Oxamil & Carbamato & I & 1 & Sí \\
\hline Javelin & Bacillus thuringiensis & Biológico & IV & 11 & No \\
\hline
\end{tabular}

${ }^{1}$ NOM-232-SSA1-2009-Plaguicidas (SSA 2018); ${ }^{2}$ modos de acción de la IRAC (Comité de Acción contra la Resistencia a Insecticidas 2019): 1) inhibidores de la acetilcolinesterasa, 3) moduladores del canal de sodio, sistema nervioso, 4) moduladores del receptor nicotínico de la acetilcolina, 5) moduladores alostéricos del receptor nicotínico de la acetilcolina, 11) disruptores microbianos de las membranas digestivas de insectos, 28). afecta al sistema nervioso y muscular.

PAP: plaguicidas altamente peligrosos (Pesticides Action Network [PAN 2019]).

95 picos con tiempos de retención, los cuales indican presencia de residuos de los siguientes plaguicidas: imidacloprid (19 muestras, $20 \%$ ), boscalid (18 muestras, $18.9 \%$ ), penconazole (15 muestras, $15.8 \%$ ), thiametoxan (12 muestras, $12.6 \%$ ), metamidofos (nueve muestras, $9.4 \%$ ), propamocarb (ocho muestras, $8.4 \%$ ), dimetoato (siete muestras, $7.3 \%$ ), ometoato (tres muestras, $3.2 \%$ ), metalaxil (dos muestras, $2.1 \%$ ) y carbofurano (dos muestras, $2.1 \%)$. Las muestras con residuos presentaron uno $(1.3 \%)$, dos $(16.2 \%)$, tres $(17.6 \%)$, cuatro $(8.1 \%)$ y cinco $(5.4 \%)$ residuos, lo cual puede atribuirse a la aplicación de mezclas de plaguicidas o bien a que no se respetan los intervalos de confianza (tiempo entre la aplicación del plaguicida y la cosecha del producto). En el cuadro IV se muestran los tiempos de retención, el porcentaje de recuperación, la desviación estándar y los límites de detección y cuantificación de los residuos de plaguicidas encontrados, así como su clasificación mediante la lista actualizada de plaguicidas altamente peligrosos de Pesticides Action Network International (PAN 2019). De los 11 plaguicidas encontrados, siete están incluidos en dicha lista.

Los tiempos de retención indican el momento en que se presentó el pico de respuesta para cada

CUADRO IV. TIEMPOS DE RETENCIÓN, PORCENTAJE DE RECUPERACIÓN Y DESVIACIÓN DEL MÉTODO DE ANÁLISIS UTILIZADO PARA EL MONITOREO DE BRÓCOLI*.

\begin{tabular}{|c|c|c|c|c|}
\hline \multirow[t]{2}{*}{ Compuesto } & \multirow{2}{*}{$\begin{array}{l}\text { Tiempo de } \\
\text { retención (min) }\end{array}$} & Recuperación & Desviación & \multirow{2}{*}{$\begin{array}{c}\text { Clasificación } \\
\text { PAP }\end{array}$} \\
\hline & & \multicolumn{2}{|c|}{$(\%)$} & \\
\hline Boscalid & 2.73 & $93.6-107.9$ & $-6.4-7.9$ & No \\
\hline Carbofuran & 2.13 & $94.9-107.7$ & $-5.1-7.7$ & $\mathrm{Si}$ \\
\hline Clorpirifos & 3.40 & $70.0-120.0$ & $-12.6-21.3$ & $\mathrm{Si}$ \\
\hline Dimetoato & 1.56 & $94.6-104.7$ & $-5.4-4.7$ & $\mathrm{Si}$ \\
\hline Imidacloprid & 1.45 & $94.6-107.9$ & $-5.4-7.9$ & $\mathrm{Si}$ \\
\hline Metamidofos & 0.59 & 93.4-106.4 & $-6.6-6.4$ & $\mathrm{Si}$ \\
\hline Metalaxil & 2.43 & $95.8-105.8$ & $-4.2-5.8$ & No \\
\hline Ometoato & 0.85 & $88.9-121.3$ & $-11.1-21.3$ & $\mathrm{Si}$ \\
\hline Penconazole & 3.02 & $81.0-126.1$ & $-9.7-26.7$ & No \\
\hline Propamocarb & 0.68 & $96.7-107.3$ & $-3.3-7.3$ & No \\
\hline Thiametoxan & 1.22 & $87.5-121.3$ & $-12.6-21.3$ & SI \\
\hline
\end{tabular}

*Límite de detección: 0.0011, límite de cuantificación: 0.0022.

PAP: plaguicidas altamente peligrosos (Pesticides Action Network [PAN 2019]). 
molécula de plaguicida. El límite refiere la cantidad más pequeña del analito en una muestra que puede ser detectada con un nivel de confianza determinado, pero no necesariamente cuantificada (SENASICA 2016); comúnmente se expresa como concentración traza del analito. Para las muestras analizadas se estableció como límite de cuantificación la mitad de la concentración del nivel 1 de la curva de calibración.

La figura 2 muestra el cromatograma de una muestra de brócoli libre de plaguicidas o blanco. La figura 2a corresponde al cromatograma de cuantificación y la $\mathbf{2 b}$ a la confirmación. Se observan otros picos de respuesta, ninguno de los cuales corresponde a tiempos de retención de plaguicidas, por lo cual se atribuyen a otras moléculas como pigmentos o sustancias contenidas en la matriz de análisis (brócoli). La figura 3a, c muestra el cromatograma de cuantificación y confirmación, respectivamente, de una muestra blanco de brócoli fortificada con imidacloprid. Por su parte, en la figura $\mathbf{3 b}$, $\mathbf{d}$ se puede apreciar, en el cromatograma de cuantificación y confirmación de la muestra 37, respectivamente, un pico bien definido que aparece en el mismo tiempo de retención que en la muestra fortificada. Carneiro et al. (2013) reportan los mismos valores en los tiempos de retención para imidacloprid.

\section{Cuantificación de residuos}

La cuantificación de residuos de plaguicidas encontrados en las muestras analizadas, en orden de

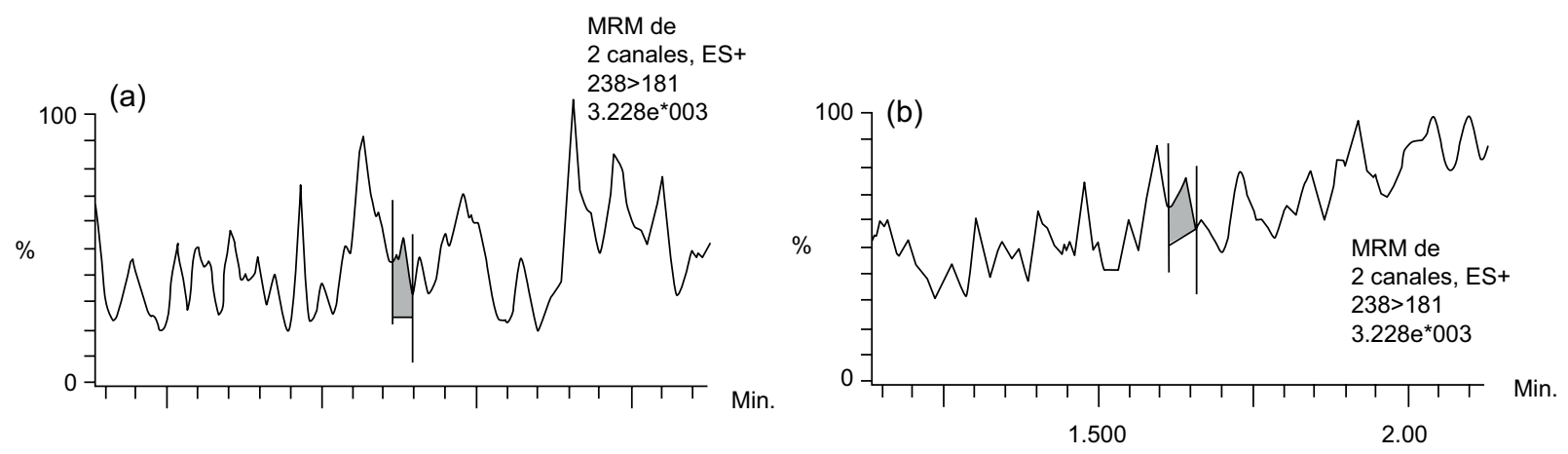

Fig. 2. Cromatogramas del blanco para brócoli utilizado para el análisis. (a) Cromatograma de cuantificación; (b) cromatograma de confirmación. MRM = Monitoreo de reacciones múltiples; Es = Electrospray.
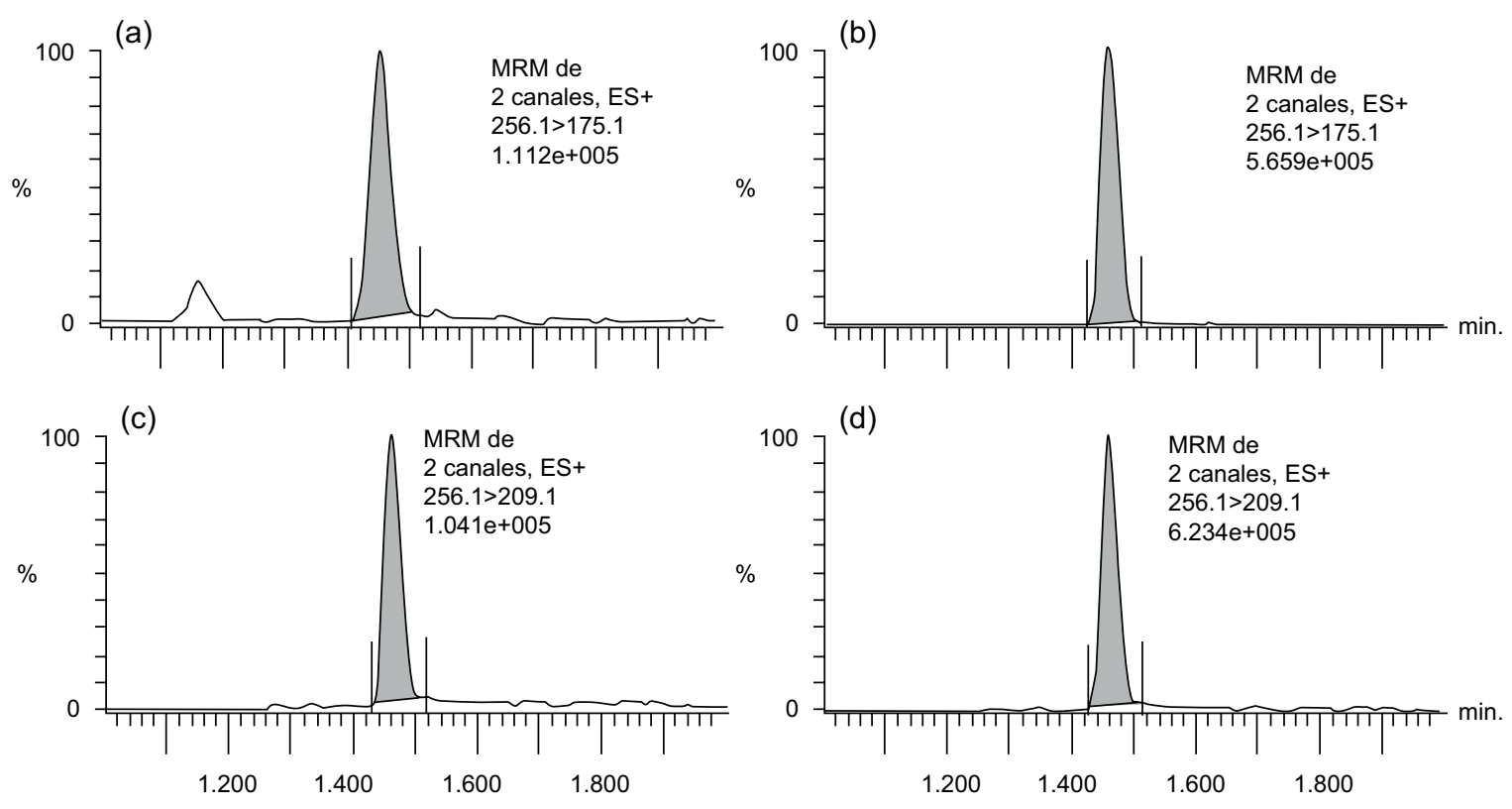

Fig. 3. (a) Cromatograma de muestra blanco fortificada con imidacloprid; (b) cromatograma de muestra 37; (c, d) confirmación de ambos cromatogramas. MRM = Monitoreo de reacciones múltiples; ES = Electrospray. 
CUADRO V. CONCENTRACIONES MEDIAS DE LOS COMPUESTOS ENCONTRADOS.

\begin{tabular}{llcccc}
\hline Plaguicida & Grupo químico & $\begin{array}{c}\text { Promedio de } \\
\text { residuos }\end{array}$ & $\begin{array}{c}\text { LMR } \\
\text { global }\end{array}$ & $\begin{array}{c}\text { LMR } \\
\text { (CICOPLAFEST) }\end{array}$ & $\begin{array}{c}\text { LMR } \\
\text { (Códex alimentarius) }\end{array}$ \\
\cline { 3 - 6 } & & & & $\mathrm{mg} / \mathrm{kg}$ \\
Propamocarb & Carbamato & 0.0061 & - & - & 3 \\
Boscalid & Estrobirulina & 0.0061 & 3 & - & - \\
Imidacloprid & Neonicotinoide & 0.0087 & - & 3.5 & - \\
Thiametoxan & Neonicotinoide & 0.0045 & - & - & 0.2 \\
Dimetoato & Organofosforado & 0.0068 & 2 & 2 & 0.01 \\
Ometoato & Organofosforado & 0.0111 & - & - & - \\
Metamidofos & Organofosforado & 0.0266 & 3.5 & 1 & - \\
Penconazole & Triazol & 0.0039 & - & - & \\
\hline
\end{tabular}

importancia y considerando la concentración media, se muestra en el cuadro V. De las muestras con presencia de residuos reportadas en el apartado anterior, $36(46.2 \%)$ y $14(18 \%)$ presentaron concentraciones no cuantificables (trazas), en tanto que 22 presentaron al menos un residuo de plaguicida (Fig. 4). Ninguna de las concentraciones de residuos de plaguicidas encontradas supera los LMR establecidos por el Codex Alimentarius, la EPA o CICOPLAFEST (2004). Sin embargo, no se encontraron los LMR para dos de los plaguicidas en ninguna de las fuentes, lo que indica que el producto no está recomendado para el cultivo de brócoli, o bien carece de LMR. Por ello el Grupo Técnico de Trabajo sobre Plaguicidas (INECC et al. 2019) señala que es necesario identificar los cultivos y productos en los que se dispone de LMR, y establecer los LMR en los cultivos y productos faltantes, en suelo, agua y aire.

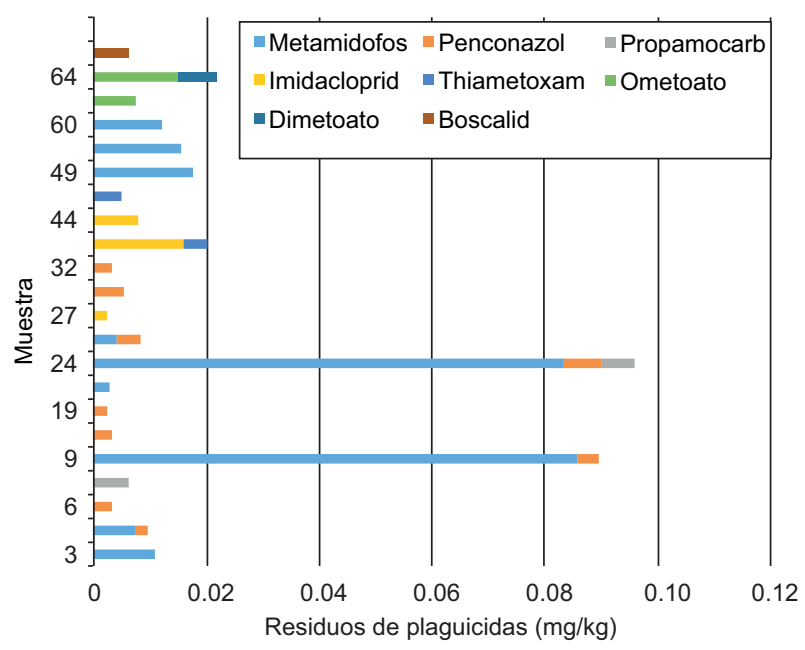

Fig. 4. Contenido de residuos de plaguicidas en las muestras de brócoli analizadas.
El análisis de la presencia de residuos de plaguicidas se ha convertido en una herramienta útil para dictaminar la inocuidad de los productos y evitar afectaciones a la salud de los consumidores. Los resultados indican que las concentraciones de residuos de plaguicidas considerados de manera individual no superan los límites establecidos por SENASICA y la EPA; sin embargo, es importante considerar que la presencia simultánea de varios residuos de plaguicidas puede generar resistencia en las plagas, así como riesgos potenciales para la salud y el ecosistema, por lo que es necesario estudiar más el efecto aditivo de los residuos.

\section{CONCLUSIONES}

Las muestras de brócoli analizadas provenientes de Puebla y Guanajuato presentaron características de calidad física (diámetro ecuatorial medio, peso y coloración de cabezuela de verde oscuro a azul verde oscuro) adecuadas a las exigencias de tamaño y color establecidas en las normas para el mercado nacional e internacional.

De las 78 muestras analizadas, el $53 \%$ (42 muestras) no presentó residuos de plaguicidas, el $18 \%$ (14 muestras) presentó concentraciones no cuantificables (trazas) y el $28 \%$ (22) presentó al menos un residuo de plaguicida. Las concentraciones de los plaguicidas encontrados en las 22 muestras no rebasaron los LMR establecidos en las normas nacionales e internacionales.

\section{REFERENCIAS}

Anastassiades M., Lehotay S.J., Stajnbaher D. y Schenck F.J. (2003). Fast and easy multiresidue method em- 
ploying acetonitrile extraction/partitioning and "dispersive solid-phase extraction" for the determination of pesticide residues in produce. Journal of AOAC International 86 (2), 412-431. https://doi.org/10.1093/ jaoac/86.2.412

Bujanos M., Marín J., Díaz E., Gámez V., Ávila P., Herrera V., Dorantes G. y Gámez V. (2013). Manejo integrado de la palomilla dorso de diamante Plutella xylostella (L.) (Lepidoptera: yponomeutidae) en el Bajío, México. INIFAP-SAGARPA, 36 pp.

Cantwell M. y Suslow T. (2007). Indicadores básicos del manejo postcosecha de brócoli. Postharvest Technology. Universidad de California, Davis, EUA, 4 pp. [en línea]. http://postharvest.ucdavis.edu/Commodity_Resources/Fact_Sheets/Datastores/Vegetables_ Spanish/?uid $=5 \& d s=803$ 22/05/2020

Carneiro R., Oliveira F., Madureira F., Silva G., de Souza W y López R. (2013). Development and method validation for determination of 128 pesticides in bananas by modified QuEChERS and UHPLC-MS/MS analysis. Food Control 33 (2), 413-423. https://doi. org/10.1016/j.foodcont.2013002.027

CEN (2009). EN 15662:2008-Foods of plant origin. Determination of pesticide residues using GC-MS and/ or LC-MS/MS following acetonitrile extraction/partitioning and clean-up by dispersive SPE-QuEChERSmethod. Comité Europeo de Normalización, Bruselas, Bélgica [en línea]. https://standards.globalspec.com/ std/10387667/EN\%2015662

CICOPLAFEST (2004). Catálogo oficial de plaguicidas. Comisión Intersecretarial para el Control del Proceso y Uso de Plaguicidas, Fertilizantes y Sustancias Tóxicas, México, 81-84 [En línea]. https://higieneambiental. com/control-de-plagas/irac-actualiza-su-clasificaciondel-modo-de-accion-de-insecticidas-y-acaricidas 20/05/2020.

Comité de Acción contra la Resistencia a Insecticidas. (2019). Folleto de clasificación del modo de acción de insecticidas y acaricidas. Versión actualizada en enero 2019 basada en la $6^{\text {a }}$ edición de IRAC Internacional. IRAC, España, 24 pp.

EPA (2019) Pesticide Tolerance Crop Grouping Revisions. US Environmental Protection Agency [en línea] https://www.epa.gov/pesticide-registration/pesticidetolerance-crop-grouping-revisions 20/08/2019

Fernández-Cevada J. y Vázquez-Ortiz J. (2003). Copitarsia incommoda (Walker) en cultivos agrícolas de exportación para el estado de Puebla. En Simposio Nacional sobre Copitarsia incommoda. Sociedad Mexicana Entomología, Colegio de Postgraduados, 29-35.

Fernández M., Fernández A., Lozano M. y Ayuso M. (2013). Different postharvest strategies to preserve broccoli quality during storage and shelf life: Controlled atmosphere and 1-MCP. J. Food Chem. 138 (1), 564-573. https://doi.org/10.1016/j.foodchem.2012.09.143

Ferrer J., García F., Reyes A. y Fernández A. (2005). Identification and quantitation of pesticides in vegetables by liquid chromatography time-of-flight mass spectrometry. TrAC Trends in Anal. Chem. 24 (7), 671-682. https://doi.org/10.1016/j.trac.2005.04.004

Fraire M., Nieto D., Cárdenas E., Gutiérrez G., Bujanos R. y Vaquera H. (2010). Efecto de variedades y densidad de plantación en la calidad física del florete de brócoli (Brassica oleracea var. italica). Revista Fitotecnia Mexicana 33 (2), 141-147.

Garay A. y Chávez C. (2014). La horticultura en México: una primera aproximación al estudio de su competitividad. Inceptum, Revista de Investigación en Ciencias de la Administración 7 (12), 271-294.

Gnanasekharan V., Shewfelt L. y Chinnan S. (1992). Detection of color changes in green vegetables. J. Food Sci. 57 (1), 149-154. https://doi. org/10.1111/j.1365-2621.1992.tb05444.x

INECC et al. 2019. Elementos para desarrollar una estrategia integral para la gestión responsable de plaguicidas en México. INECC/ONU Medio Ambiente/OPS/OMS/ DGGIMAR-SEMARNAT/-DGPSSecretaría de Salud/STCONAPRA-Secretaría de Salud/RETOMEX, Ciudad de México, 47 pp. [en línea] https://www.gob.mx/cms/uploads/attachment/ file/451603/Elementos_para_Desarrollar_una_Estrategia_Integral_de_Manejo_Responsable_de_ Plaguicidas_final_3_.pdf 06/05/2020

Jaga K. y Dharmani C. (2005). The epidemiology of pesticide exposure and cancer: A review. J. Environ. Health 20 (1), 15-38. https://doi.org/10.1515/ REVEH.2005.20.1.15

Lagunes-Tejeda A. y Villanueva-Jiménez J.A. (1994). Toxicología y manejo de insecticidas. Colegio de Postgraduados, Montecillos, Estado de México, 264 pp.

Marín A. y Bujanos R. (2001). Insectos plaga de brócoli y coliflor y sus enemigos naturales en la región del Bajío, México. Publicación especial número 2. INIFAP, 26 pp.

Martínez M., Leiva A., Sotomayor C., Victoriano T., von Chrismar A. y Pineda S. (2012). Factores de riesgo cardiovascular en estudiantes de la Universidad Austral de Chile. Rev. Méd. Chile 140 (4), 426-435. https://doi. org/10.4067/S0034-98872012000400002

Obare S., de Chandrima G., Haywood T., Samuels T., Adams C., Masika N., Murray D., Anderson G., Campbell K. y Fletcher K. (2010). Fluorescent chemosensors for toxic organophosphorus pesticides: a review. Sensors Basel 10 (7), 7018-7043. https://doi.org/10.3390/ s100707018 
Ortiz F., López M., Fernández M., Yruela C., Navas A., López J., Lirola J. y Caballero E. (2003). Aplicación de plaguicidas. Nivel cualificado. 2a ed. Junta de Andalucía-Consejería de Agricultura y Pesca. Andalucía, España, 235 pp.

PAN (2019). List of highly hazardous pesticides. PAN International, Hamburgo, Alemania, 47 pp. [en línea]. https://pan-international.org/wp-content/uploads/ PAN_HHP_List.pdf 02/04/2020.

Pérez M., Jiménez S., Ebrahimzadeh A., Verdejo M., Chaves A. y Lao T. (2008). Propuesta de una metodología para la determinación del color del tejido vegetal. Actas de Horticultura No. 52. Innovación y Futuro en la Jardinería: I Simposio Iberoamericano de Horticultura Ornamental. Pontevedra, España [en línea]. https://docplayer.es/69406713-Actas-de-horticultura.html 12/11/2019.

Pérez A., Segura A., García R., Colinas T., Pérez M., Vázquez A. y Navarro H. (2009). Residuos de plaguicidas organofosforados en cabezuela de brócoli (Brassica oleracea). Determinación por cromatografía de gases. Rev. Int. Contam. Amb. 25 (2), 103-110.

Pérez A., García R., Pérez M. y Navarro H. (2014). Sistema de producción y parámetros de calidad agronómica de brócoli en Mixquic, DF. Rev. Mex. de Cienc. Agr. 5 (8), 1459-1468.

Rocha J. y Cisneros D. (2019). La producción de brócoli en la actividad agroindustrial en México y su competitividad en el mercado internacional. Acta Universitaria 29 (1), 1-13. https://doi.org/10.15174/au.2019.2156

Royal Horticultural Society (2007). Colours of the Royal Horticultural Society. Colour charts edition V [en línea]. www.rhscf.orgfree.com 05/08/2019.

SE (2005). Pliego de condiciones pc-034-2005 para el uso de la marca oficial México Calidad Suprema en brócoli. Secretaría de Economía. Diario Oficial de la Federación, 5 de julio [en línea]. http://dof.gob.mx/ nota_detalle_popup.php? $\operatorname{codigo}=204391008 / 08 / 2019$

SENASICA (2016). Criterios de funcionamiento de métodos analíticos. Servicio Nacional de Sanidad, Inocuidad y Calidad Agroalimentaria [en línea]. https://www. gob.mx/cms/uploads/attachment/file/128467/CriteriosdeFuncionamiento_abril_2016_1_.pdf 20/05/2020

SEPAFIN (1983) Norma Mexicana NMX-FF-046-1982. Productos alimenticios no industrializados para uso humano hortalizas en estado fresco brócoli. Secretaría de Patrimonio y Fomento Industrial. Diario Oficial de la Federación, 14 de enero [en línea]. http://dof. gob.mx/nota_detalle.php? $\operatorname{codigo}=4791906 \& \mathrm{fec}$ ha $=14 / 01 / 1983$ 8/18/2020
SIAP (2020). Avance de siembras y cosechas. Resumen nacional por cultivo. Servicio de Información Agroalimentaria y Pesquera [en línea]. http://infosiap.siap.gob. mx:8080/agricola_siap_gobmx/ResumenDelegacion. do $14 / 06 / 2020$.

Shewfelt L., Heaton K. y Batal M. (2006). Nondestructive color measurement of fresh broccoli. J. Food Sci. 49 (6), 1612-1613. https://doi.org/10.1111/j.1365-2621.1984. tb12857.x

Soto J. (1991). Efecto de la fertilización nitrogenada y la población sobre el rendimiento y calidad de brócoli (Brassica oleracea var. Itálica) durante dos ciclos de cargo. Agron. Costarric. 15 (1/2), 19-27.

Suárez D., Bautista N., Valdez J., Angulo A., Alatorre R., Vega J., Equihua A. y Manuel V. (2006). Fluctuación poblacional de Copitarsia decolora (Gueéne) y su asociación con crucíferas comerciales. Agrociencia 40 (4), 501-509.

SSA (2018). Modificaciones a la Norma Oficial Mexicana NOM-232-SSAI-2009. Plaguicidas. Que establece los requisitos del envase, embalaje y etiquetado de productos grado técnico y para uso agrícola, forestal, pecuario, jardinería, urbano, industrial y doméstico. Secretaria de Salud. Diario Oficial de la Federación, 18 de diciembre [en línea]. http://www.dof.gob.mx/ normasOficiales/4020/salud/salud.htm 21/08/2019.

Torres D. (2011). Caracterización del sistema de producción de brócoli y efecto de la fertilización órgano mineral en la dinámica del nitrógeno. Tesis de Maestría en Estrategias para el desarrollo agrícola regional. Colegio de Postgraduados, Puebla, México.

Villalobos S., Castellanos Z., Tijerina L. y Crespo G. (2005). Efecto de la tensión de humedad en el suelo sobre rendimiento y calidad del brócoli con riego por goteo. Terra Latinoamericana, 23 (3), 321-328.

Weichenthal S., Moase C. y Chan P. 2010. A Review of pesticide. Exposure and cancer incidence in the agricultural health study. Cohort. J. Environ Health Perspectives 118 (8), 1117-1125. https://doi.org/10.1289/ ehp.0901731

Westcott M. y Callan N. 1990. Modeling plant population and rectangularity effects on broccoli head weights and yield. J. Amer. Soc. Hort. Sci. 115 (6), 893-897. https:// doi.org/10.21273/JASHS.115.6.893

Wigle T., Arbuckle E., Walker M., Wade G., Liu L. y Krewski D. (2007). Environmental hazards: Evidence for effects on child health. J. Tox. Environ. Health B 10 (12), 3-39. https://doi.org/10.1080/10937400601034563 